\title{
Crenças de mães de diferentes níveis socioeconômicos sobre punição física e privação de privilégios
}

\section{Beliefs of mothers from different socioeconomic levels about physical punishment and deprivation of privileges}

Creencias de madres de diferentes niveles socioeconómicos
acerca de castigo físico y privación de privilegios

\section{Paulo Henrique Barbosa do Carmo*}

Faculdade Anísio Teixeira - Feira de Santana, Bahia, Brasil

\author{
Patrícia Alvarenga** \\ Universidade Federal da Bahia - UFBA, Salvador, Bahia, Brasil
}

Taiane Costa de Souza Lins***

Universidade Federal da Bahia - UFBA, Salvador, Bahia, Brasil

\begin{abstract}
RESUMO
Este estudo comparou as crenças de mães de diferentes níveis socioeconômicos sobre dois tipos de práticas de socialização coercitivas: a punição física e a privação de privilégios. Participaram 40 mães com filhos entre cinco e seis anos, de ambos os sexos. Vinte mães eram de nível socioeconômico baixo e 20 mães eram de nível socioeconômico médio/alto, de acordo com os critérios de Hollingshead. As mães responderam a uma entrevista estruturada de crenças sobre as práticas coercitivas, e suas respostas foram submetidas à análise de conteúdo. A única diferença significativa entre os dois grupos mostrou que as mães de nível socioeconômico médio/alto apresentaram maior frequência de crenças na eficácia da privação de privilégios. Discute-se os possíveis efeitos da ampla difusão das desvantagens da punição física e de outras práticas coercitivas sobre as crenças maternas.
\end{abstract}

Palavras-chaves: mães, crenças, socialização, punição, privação.

\begin{abstract}
This study compared the beliefs of mothers from different socioeconomic levels about two types of coercive socialization practices: physical punishment and deprivation of privileges. Participants in the study were 40 mothers of children of both sexes, aged 5 to 6 years. Twenty mothers belonged to low socioeconomic status, and twenty mothers belonged to medium/high socioeconomic status according to Hollingshead. The mothers answered a structured interview regarding beliefs about coercive practices, and their answers were submitted to content analysis. The only significant difference between the two groups showed that mothers from middle/high
\end{abstract}


socioeconomic status had a higher frequency of beliefs in the efficacy of deprivation of privileges. The possible effects of the widespread debate about the disadvantages of physical punishment and other coercive practices on maternal beliefs are discussed.

Keywords: mothers, beliefs, socialization, punishment, privileges.

\section{RESUMEN}

Este estudio comparó las creencias de madres de diferentes niveles socioeconómicos sobre dos tipos de prácticas de socialización coercitivas: el castigo físico y la privación de privilegios. Las participantes fueron 40 madres con niños entre cinco y seis años, de ambos sexos. Veinte madres eran de bajo nivel socioeconómico y 20 madres eran de nivel socioeconómico medio/alto, de acuerdo con los criterios de Hollingshead. Las madres respondieron una entrevista estructurada sobre las creencias sobre las prácticas coercitivas, y sus respuestas fueron sometidas a análisis de contenido. La única diferencia significativa entre los dos grupos mostró que las madres de nivel socioeconómico medio/alto tenían una frecuencia más alta de creencias en la eficacia de la privación de privilegios. Se discute los posibles efectos de la difusión del debate sobre las desventajas del castigo físico y otras prácticas coercitivas sobre las creencias maternas.

Palabras clave: madres, creencias, socialización, castigo, punición.

\section{Introdução}

As crenças parentais constituem um importante foco de interesse para pesquisadores dedicados ao estudo das relações entre a parentalidade e o desenvolvimento infantil. Embora ações ou comportamentos direcionados à criança, tais como as práticas de socialização, sejam investigados com maior frequência, é consensual a noção de que esses comportamentos e práticas estejam relacionados a ideias ou crenças. Assim, as crenças parentais podem ser definidas como conjuntos de ideias implícitas nos julgamentos, escolhas e decisões dos pais, que funcionam como modelos ou roteiros de ação para o alcance de objetivos relacionados à criação ou socialização dos filhos (Kobarg, Sachetti, \& Vieira, 2006). Desse modo, a compreensão das relações entre a parentalidade e o desenvolvimento infantil depende de uma concepção dinâmica e integrada desses fenômenos, que articule aspectos como crenças, valores e metas às ações ou práticas (Kobarg et al., 2006; Moura et al., 2004).

As práticas e as crenças parentais sofrem influência de inúmeros fatores relacionados ao contexto socioeconômico e cultural em que a família está inserida, assim como às características particulares dos pais e dos filhos e de suas histórias de vida. A noção de nível socioeconômico (NSE) possibilita o agrupamento de alguns desses fatores. Para Ribas Jr., Moura, Soares, Gomes e Bornstein (2003), o NSE se refere à distribuição não uniforme de valores sociais, como educação, prestígio social e renda, entre indivíduos, famílias ou 
grupos em um sistema social desigual, que pode contribuir para o desenvolvimento de perspectivas diferentes a respeito de indicadores sociais, como a ocupação, a educação, a saúde, entre outros. Apesar de discordâncias, o NSE é multidimensional, sendo frequentemente medido e avaliado através de variáveis como o nível de escolaridade, a ocupação e a renda dos participantes do estudo ou da família (Bornstein \& Bradley, 2003; Ribas Jr., Moura, Soares, et al., 2003).

Embora não exista na literatura, um consenso sobre o que precisamente o NSE representa, há evidências de que crianças de camadas sociais mais favorecidas do ponto de vista socioeconômico, avaliado, em especial, pelo envolvimento da família em atividades acadêmicas e intelectuais, por ocupações mais especializadas e com maior prestígio social, e pela renda, possuem maior acesso a recursos que favorecem o desenvolvimento pleno, quando comparadas a crianças de NSE baixo (Knutson, DeGarmo, Koeppl, \& Reid, 2005; Ribas Jr., Moura, \& Bornstein, 2003). Entre esses recursos, destacam-se aqueles referentes à parentalidade, como por exemplo, as práticas de socialização. Por outro lado, parece ser consenso na literatura da Psicologia do Desenvolvimento que as situações adversas relacionadas às dificuldades econômicas, tais como o estresse, 0 desemprego e a fome, tendem a aumentar a probabilidade do emprego de práticas pouco contingentes e pouco consistentes, e das chamadas estratégias coercitivas, como a punição física e a privação de privilégios (Alvarenga, Magalhães, \& Gomes, 2012; Carmo, 2009; Friedson, 2016; Mistry, Vandewater, Huston, \& McLoyd, 2002; Theunissen, Vogels, \& Reijneveld, 2015).

A punição física envolve o uso de ações que tendem a causar dor nas crianças, tais como bater, beliscar, puxar, sacudir a criança, entre outras. A privação de privilégios se refere às estratégias que impedem a criança de utilizar algo ou de se envolver em atividades que sejam interessantes para ela, como assistir televisão, brincar, comer doces ou guloseimas, entre outros (Alvarenga et al., 2012). A literatura mostra claramente que o uso frequente de práticas de socialização coercitivas está relacionado a diferentes tipos de problemas de comportamento em crianças, entre eles, a agressividade e o comportamento desafiador (Alvarenga \& Palma, 2012; Alvarenga \& Piccinini, 2001; Bayer et al., 2012; Lins, Alvarenga, Paixão, Almeida, \& Costa, 2012; Weber, Viezzer, \& Brandenburg, 2004), o que evidencia a relevância de estudos que investiguem os determinantes desse tipo de prática e as crenças a elas associadas.

A literatura da Psicologia do Desenvolvimento apresenta evidências mais consistentes no que se refere ao uso da punição física por pais de baixo NSE, porém, pouco se sabe ainda sobre a concessão ou a privação de privilégios (Theunissen et al., 2015). Carmo e Alvarenga (2012), ao comparar o uso de práticas coercitivas por mães de 
crianças entre cinco e seis anos, de diferentes níveis socioeconômicos da cidade de Salvador/Brasil, avaliados através do nível de escolaridade, da ocupação e do estado civil, verificaram que as mães de NSE baixo relataram, em média, mais uso da punição física em comparação às mães de NSE médio/alto. Nesse mesmo sentido, Alvarenga et al. (2012), em um estudo com o objetivo de investigar as relações entre as práticas de socialização maternas e os problemas externalizantes, com 64 díades mãe-criança, residentes também na cidade de Salvador/Brasil, verificaram que quanto menor a renda, assim como a escolaridade das mães, mais frequente foi o relato do uso da punição física. Jansen et al. (2012) encontraram resultados semelhantes ao avaliarem os determinantes sociodemográficos, psicossociais do uso da disciplina severa por pais e mães de 3756 crianças com três anos de idade que viviam em uma cidade holandesa. De acordo com os achados, o baixo NSE das famílias, avaliado através das dificuldades financeiras e do nível de escolaridade, esteve significativamente associado ao uso de punição física (dar beliscões e sacudir a criança) por parte das mães, embora não dos pais. Grogan-Kaylor e Otis (2007), por outro lado, ao avaliar a prevalência e a cronicidade do uso de estratégias que envolvem a punição física em uma amostra representativa de pais norteamericanos, não verificaram associação entre viver em condições de pobreza (comparação entre o rendimento da família durante o ano com os indicadores de pobreza para aquele ano fornecidos pelo Censo dos EUA) e o uso de punição física.

Com relação à privação de privilégios, o número de estudos a esse respeito é limitado e as poucas investigações realizadas têm apresentado resultados menos consistentes. Carmo e Alvarenga (2012) não verificaram nenhuma diferença nos relatos de mães de NSE baixo e médio/alto sobre o uso desse tipo de prática. De modo semelhante, Socolar, Savage, Keyes-Elstein e Evans (2005), ao examinarem fatores que influenciam as práticas utilizadas especialmente por mães norte-americanas para disciplinar os comportamentos dos filhos entre 12 e 19 meses de vida, não encontraram relação significativa entre a privação de privilégios e fatores de risco social da família, como baixa renda, baixo nível de escolaridade, desemprego e ser imigrante. Por outro lado, de acordo com os achados de Alvarenga et al. (2012), quanto maior a renda familiar, maior foi o uso de privação de privilégios e castigos, como deixar a criança sentada no sofá ou sozinha em uma parte da casa.

Segundo Lerner (2003) e Bradley e Corwyn (2002), ainda não estão claros os mecanismos através dos quais o NSE poderia repercutir nesses processos. Uma hipótese investigada na literatura é a de que a relação entre o NSE e as práticas de socialização parentais possam ser mediadas pelas crenças e pelos valores dos pais a respeito de tais práticas (Bor \& Sanders, 2004; Doe, 2000; Passini, Pihet, \& Favez, 
2014). Para Shor (2000) e Giles-Sims e Lockhart (2005), as crenças parentais sobre a eficácia e a legitimidade do uso de práticas coercitivas seriam construídas em função do NSE. Assim, quanto menor o NSE da família mais legítimo e funcional o uso da coerção seria considerado pelos pais e mais frequente tenderia a ser o uso desse tipo de estratégia. Nessa mesma perspectiva, Bem e Wagner (2006) relataram uma série de estudos realizados por Kohn em meados dos anos 1970, em que o autor relaciona os valores parentais ao NSE, avaliado através, por exemplo, da escolaridade, da ocupação e da renda. Os estudos desse pesquisador demonstraram que pais pertencentes a NSE mais elevados tenderiam a priorizar valores de autodireção nas crianças (ex. autocontrole, responsabilidade, curiosidade), enquanto famílias de NSE mais baixos se preocupariam mais com valores de conformidade (ex. limpeza, bons modos, obediência). Para o autor, os valores relacionados à obediência poderiam explicar, pelo menos em parte, o uso mais frequente da punição por famílias de baixo NSE.

Cappa e Khan (2011) avaliaram, em 34 países dos continentes africano, americano, asiático e europeu, a variabilidade nas crenças maternas e as práticas utilizadas pelas próprias mães (ou por outros cuidadores) de crianças e adolescentes entre dois e 14 anos. De acordo com os resultados do estudo, mães com menor nível de escolaridade, na maioria dos países, acreditavam que a punição física era uma estratégia necessária para regular o comportamento dos filhos. Além disso, em 20 dos 34 países, verificou-se que em famílias com rendas mais baixas, as mães acreditavam que a punição física era uma estratégia necessária. Os achados do estudo mostram ainda que em 32 países é mais provável punir fisicamente crianças e adolescentes quando a mãe acredita que esse tipo de prática é necessário.

Shor (2000), em um estudo sobre as diferenças na percepção parental das práticas entre famílias residentes em bairros de renda baixa e média da cidade de Jerusalém, verificou que as práticas de socialização adotadas por pais que moravam no bairro de baixa renda eram mais coercitivas em comparação às práticas adotadas por pais que moravam no bairro de renda média, e que os primeiros acreditavam mais na legitimidade do uso da punição física na regulação do comportamento dos filhos, do que os pais de renda média. O autor ressalta ainda que as crenças parentais sobre a legitimidade e a eficácia do uso de práticas coercitivas diferiram muito entre os dois grupos. Os pais com menor renda enfatizaram a obrigatoriedade da obediência dos filhos aos pais, demonstraram valores autoritários e destacaram a necessidade de tais práticas para manter as crianças em segurança, já que moravam em bairros perigosos. Por outro lado, os pais com renda média não compartilhavam essa preocupação, eram contra o uso da punição 
física, e demonstravam valores mais democráticos dentro das famílias.

Algumas evidências e hipóteses teóricas da literatura indicam relações entre o NSE e as crenças e os valores familiares sobre a criação e a socialização dos filhos no contexto familiar. Outros estudos, por sua vez, mostram que essas crenças e valores podem mediar a relação entre o NSE e as práticas que são efetivamente adotadas pelos pais na interação com suas crianças. Contudo, no Brasil, um país com marcante estratificação e desigualdade social, são poucos os estudos que examinam questões relacionadas a essa problemática. Soma-se a isso a escassez de estudos sobre a privação de privilégios, uma prática de socialização ainda pouco investigada na literatura nacional e internacional. Nesse sentido, o presente estudo comparou as crenças de mães de nível socioeconômico baixo (NSE-B) com as de mães de nível socioeconômico médio/alto (NSE-M) sobre dois tipos de práticas de socialização coercitivas: a punição física e a privação de privilégios. A hipótese foi de que mães de NSE-B relatariam mais crenças favoráveis à punição física e à privação de privilégios quando comparadas às mães de NSE-M.

\section{Método}

\subsection{Participantes}

Participaram do estudo 40 mães, casadas ou em união estável, com idade entre 25 e 45 anos ( $M=33,2$ e $D P=6,6)$, e com filhos entre cinco e seis anos de idade. Todas as participantes faziam parte de um estudo mais amplo (Carmo, 2009) que foi aprovado por um Comitê de Ética em Pesquisa e está de acordo com as normas éticas de conduta para pesquisas com humanos. Foram constituídos dois grupos de mães: (1) NSE-B: grupo formado por 20 mães de nível socioeconômico baixo; (2) NSE-M: grupo formado por 20 mães de nível socioeconômico médio/alto. As crianças, 20 meninos e 20 meninas, a respeito das quais as mães responderam as entrevistas, foram igualmente distribuídas quanto ao sexo nos dois grupos investigados. As mães foram contatadas através de uma escola pública e uma escola privada da cidade de Salvador.

A classificação do nível socioeconômico das famílias foi feita a partir dos critérios propostos por Hollingshead (1975) adaptados por Tudge e Frizzo (2002). Esta escala avalia o nível socioeconômico através de quatro fatores: educação, ocupação, estado civil e sexo. Detalhes sobre as características sociodemográficas dos dois grupos são apresentados na Tabela 1. 
Tabela 1

Caracteristicas Sociodemográficas das Participantes

\begin{tabular}{|c|c|c|c|c|}
\hline & $\begin{array}{c}\text { Grupo NSE-B } \\
(\mathrm{n}=20) \\
M(D P)\end{array}$ & $\begin{array}{c}\text { Grupo NSE-M } \\
(\mathrm{n}=20) \\
M(D P)\end{array}$ & & \\
\hline \multicolumn{5}{|l|}{ Idade da criança (anos) } \\
\hline$M$ & 5,20 & 5,50 & $t=2,04$ & $p=0,04$ \\
\hline$D P$ & 0,41 & 0,51 & & \\
\hline \multicolumn{5}{|l|}{ Idade da mãe (anos) } \\
\hline$M$ & 30,95 & 35,45 & $t=2,24$ & $p=0,03$ \\
\hline$D P$ & 5,23 & 7,26 & & \\
\hline \multicolumn{5}{|c|}{ Escolaridade da mãe (anos) } \\
\hline$M$ & 8,60 & 14,10 & $t=5,54$ & $p=0,001$ \\
\hline$D P$ & 3,71 & 2,42 & & \\
\hline \multicolumn{5}{|l|}{ Ocupação da mãe } \\
\hline Não trabalha fora & $11(55 \%)$ & $6(30 \%)$ & $x^{2}=2,55$ & $p=0,11$ \\
\hline Trabalha fora & $9(45 \%)$ & $14(70 \%)$ & & \\
\hline \multicolumn{5}{|l|}{ Número de fillhos } \\
\hline M & 1,75 & 1,90 & $t=0,63$ & $p=0,53$ \\
\hline$D P$ & 0,71 & 0,78 & & \\
\hline \multicolumn{5}{|c|}{ Número de pessoas na casa } \\
\hline$M$ & 4,35 & 3,80 & $t=1,52$ & $p=0,13$ \\
\hline$D P$ & 1,46 & 3,80 & & \\
\hline \multicolumn{5}{|l|}{ Renda Familiar (RS) } \\
\hline$M$ & 655,50 & 5.500 & $t=11,46$ & $p=0,001$ \\
\hline$D P$ & 312,48 & 1.863 & & \\
\hline
\end{tabular}

A análise com o teste $t$ de Student e com o teste de Qui-quadrado revelou algumas diferenças significativas entre os grupos NSE-B e NSE-M. Quanto à idade da criança, foi constatada uma diferença significativa ( $t=2,04 ; p=0,04)$, que indica uma maior idade das crianças do grupo NSE-M. Com a variável idade da mãe, também foi encontrada uma diferença significativa $(t=2,24 ; p=0,03)$, que indica uma maior idade das mães do grupo NSE-M. Outras diferenças significativas foram encontradas em escolaridade da mãe $(t=5,54 ; p$ $=0,001)$, indicando um maior número de anos de estudo das mães do grupo NSE-M, e em renda familiar ( $t=11,46 ; p=0,001$ ), indicando que as famílias do grupo NSE-M têm uma renda mensal maior. As diferenças encontradas na escolaridade das mães e na renda familiar haviam sido previstas por se tratarem de características relacionadas à classificação do nível socioeconômico das famílias. Nesse sentido, a discrepância entre os grupos era esperada e era condição necessária para o delineamento escolhido para o presente estudo. As diferenças na idade da criança e na idade da mãe, embora tenham sido significativas, não representam diferenças relevantes do ponto de vista psicológico, como indicam as médias apresentadas na Tabela 1. De qualquer modo, tais variáveis foram inseridas como covariantes nas análises das crenças sobre as práticas coercitivas. 


\subsection{Procedimentos}

As mães foram contatadas através de escolas da cidade de Salvador. Inicialmente as mães receberam, nas escolas, uma carta e uma ficha de contato solicitando o nome e o telefone. A carta explicava os objetivos da pesquisa e instruía quanto à devolução da ficha de contato preenchida, caso a mãe consultada optasse por participar do estudo. O primeiro contato com o pesquisador aconteceu por telefone, quando foram agendadas data e hora das entrevistas. As entrevistas ocorreram na própria escola ou na residência das participantes, dependendo da disponibilidade e da escolha de cada mãe. Como o presente estudo é parte de outro mais amplo, para este, na data, horário e local agendados, as participantes responderam à Entrevista de Crenças sobre a Coerção e à Ficha de Informações Demográficas da Família. As entrevistas foram gravadas em áudio e transcritas para análise. Destaca-se que parte dos dados e dos procedimentos deste estudo encontram-se apresentados em outro estudo (Carmo \& Alvarenga, 2012).

\subsection{Instrumentos}

Ficha de Informações Demográficas da Família. Essa ficha foi aplicada com o objetivo de serem obtidas informações demográficas da família, tais como: idade, profissão e nível de instrução dos pais, renda familiar, sexo e idade da criança. A ficha foi utilizada também para a classificação do NSE de acordo com os critérios de Hollingshead (1975).

Escala dos quatro fatores de Hollingshead (1975). A escala classifica o NSE de acordo com quatro fatores: educação, ocupação, estado civil e sexo. Multiplica-se o nível da ocupação (que pode variar de um a nove) por 5 e o nível educacional (que pode variar de um a sete) por três, e esses dois valores são somados. A escala prevê ainda outras possibilidades de arranjos familiares para o cálculo do NSE: (a) se a esposa trabalhar, soma-se o NSE da esposa e o do marido e divide-se o valor por dois; (b) se a esposa não trabalhar, o cálculo deve envolver apenas o marido; (c) caso um dos cônjuges seja divorciado e estiver recebendo pensão alimentícia do ex-cônjuge, o NSE do ex-cônjuge também deve ser contabilizado; (d) se um dos cônjuges for divorciado, trabalhar e também receber pensão alimentícia, calcula-se o NSE dos dois cônjuges e divide-se por dois; (e) se um dos cônjuges for viúvo e não trabalhar, mas receber pensão do cônjuge falecido, calcula-se o NSE a partir da ocupação e educação da pessoa falecida. A pontuação, segundo a escala, classifica os indivíduos da seguinte forma: (a) Nível 1 - trabalhadores sem habilidades (ex. empregados domésticos); (b) Nível 2 trabalhadores com poucas habilidades (ex. operadores de máquinas); 
(c) Nível 3 - trabalhadores com habilidades (ex. auxiliares de escritório, balconistas); (d) Nível 4 - proprietários de empresas de médio porte, técnicos; (e) Nível 5: - proprietários de grandes empresas e profissionais de nível superior. Este instrumento não oferece um protocolo para a coleta das informações sociodemográficas das famílias, caracterizando-se unicamente como um conjunto de critérios definidos para a classificação do NSE. Por essa razão, foi utilizada, além da Escala de Hollingshead, a Ficha de Informações Demográficas da Família com o objetivo de coletar as informações necessárias, para a posterior aplicação dos critérios da escala. Para fins deste estudo, a classificação do NSE foi realizada da seguinte forma: os níveis 4 e 5 corresponderam ao nível socioeconômico médio/alto, e os níveis 1 e 2 corresponderam ao nível socioeconômico baixo. Os participantes que obtiveram classificação no nível 3 foram excluídos da amostra. A exclusão desses participantes visou a intensificar as diferenças entre os grupos NSE-M e NSE-B, que foram alvo do estudo.

Entrevista de Crenças Sobre a Coerção. A entrevista semiestruturada foi desenvolvida para um estudo mais amplo que deu origem à presente investigação (Carmo, 2009) e investigou o que as mães pensavam sobre o uso da coerção na regulação do comportamento infantil, sobre sua eficácia, bem como sobre as vantagens e desvantagens desse tipo de estratégia. A entrevista é composta por oito questões e é dividida em duas partes: a primeira parte contém perguntas sobre as crenças das mães a respeito do uso da punição física e seus efeitos, investigando vantagens e desvantagens dessa estratégia, por exemplo, "O que você acha do uso da palmada na educação das crianças", "Você acha que a palmada funciona?"; e a segunda parte avalia as crenças das mães a respeito do uso da privação de privilégios (castigo), investigando os mesmos pontos abordados na primeira parte da entrevista. As respostas das mães foram submetidas à Análise de Conteúdo (Bardin, 1977/2011), que gerou uma estrutura de oito categorias temáticas: (a) eficácia da punição física: relatos maternos que indicam crenças de que a punição física modifica ou reduz comportamentos inadequados ou indesejáveis da criança, incluindo referências vagas à obediência e respeito gerados por esse tipo de estratégia (ex. "Ele tem mais respeito com a gente, obedece mais"); (b) ineficácia da punição física: relatos da mãe que indicam seu descrédito em relação à punição física, ou que oferecem evidências de que a experiência da mãe com essa estratégia não produziu ou não costuma produzir mudanças nos comportamentos inadequados ou indesejáveis da criança (ex.: "Eu nunca vi nenhum tipo de mudança, nunca vi resolver nada."); (c) limites para a punição física: relatos que mostram que as mães creem que a punição física, quando utilizada com critério e com intensidade moderada, pode ser eficaz e não 
causar danos à criança (ex: “De vez em quando eu bato. Mas não pode exagerar né?"); (d) necessidade da punição física: relatos que indicam que as mães, apesar de perceberem as desvantagens da punição física, acreditam que ela seja uma estratégia necessária devido à incapacidade de compreensão da criança, ou então que esta é uma estratégia necessária em determinadas situações (ex: "A criança não tem assim um entendimento, às vezes a gente tá falando ela faz. Aí eu bato"); (e) eficácia da privação de privilégios: relatos que indicam que a mãe acredita que a privação de privilégios modifica ou reduz comportamentos inadequados ou indesejáveis da criança, incluindo referências vagas à obediência e respeito gerados por esse tipo de prática (ex: "Ele me obedece quando está de castigo"); (f) ineficácia da privação de privilégios: relatos que indicam que a participante não percebe ou não acredita no potencial da privação de privilégios para provocar mudanças em comportamentos inadequados ou indesejáveis da criança. Por vezes, as participantes comparavam esta estratégia à punição física, destacando a sua maior eficácia (ex: "Eu prefiro bater do que castigar...os castigos não adiantam"); (g) limites para a privação de privilégios: relatos que mostram que as mães creem que a privação de privilégios, assim como a punição física, também pode causar danos à criança, e que, portanto, deve ser utilizada com critério e com intensidade moderada, podendo ser eficaz (ex: "Sendo um castigo que não maltrate, acho que pode"; e (h) necessidade da privação de privilégios: relatos que sugerem que as mães acreditam que certos comportamentos infantis só podem ser regulados, ou podem ser melhor regulados através desse tipo de prática coercitiva (ex: "Brigando com o primo ou querer comer alguma coisa que não pode na hora, ai tem que ser castigo").

A estrutura das categorias foi construída com base no estudo de Guerra, Huesmann, Tolan, Van Acker e Eron (1995), porém, as categorias propostas para o presente estudo e suas definições derivaram, em grande parte, da análise do relato das participantes. As entrevistas realizadas foram submetidas à análise de conteúdo, e a classificação das crenças sobre a coerção relatadas pelas mães foi realizada por dois codificadores que receberam treinamento e que desconheciam o grupo ao qual cada mãe pertencia. $O$ índice de fidedignidade entre os codificadores foi calculado com uma amostra de $30 \%$ das entrevistas e atingiu 0,87 (Kappa) para as crenças sobre a punição física e 0,89 (Kappa) para as crenças sobre a privação de privilégios. 


\section{Resultados}

A partir da análise de conteúdo das respostas das mães à entrevista, foram identificadas 320 crenças maternas sobre a coerção das quais $52 \%$ foram relatadas pelo grupo NSE-B e $48 \%$ pelo grupo NSE-M. Do total de crenças, $56 \%$ foram crenças em relação a punição física e $44 \%$ sobre a privação de privilégios. Do total de respostas sobre a punição física, $51 \%$ foram relatadas pelo grupo NSE-B e $49 \%$ pelo grupo NSE-M. Do total de respostas sobre a privação de privilégios/castigos, $53 \%$ foram relatadas pelo grupo NSE-B e $47 \%$ pelo grupo NSE-M.

Com o objetivo de avaliar as diferenças nas frequências médias das crenças sobre a punição física entre os dois grupos, foi realizada uma Análise de Covariância (ANCOVA) com as frequências médias para cada categoria. As variáveis idade da mãe e idade da criança foram incluídas como covariantes. A Tabela 2 apresenta a média, o desvio padrão, o valor de $\mathrm{F}$ e o nível de significância nas categorias de crenças sobre a punição física nos dois grupos. Os resultados demonstraram não haver diferenças significativas nas crenças sobre a punição física mencionadas pelas mães em nenhuma das categorias.

\section{Tabela 2}

Incidência Média, Desvio Padrão, Valor de F e Nivel de Significância do Total de Crenças Maternas Sobre a Punição Fisica Mencionadas pelos Dois Grupos

\begin{tabular}{lcccc}
\hline & $\begin{array}{c}\text { Grupo NSE-B } \\
\text { Média (D.P) }\end{array}$ & $\begin{array}{c}\text { Grupo NSE-M } \\
\text { Média (D.P) }\end{array}$ & $\mathrm{F}$ & $p<$ \\
\hline Eficácia da punição fisica & $0,95(0,99)$ & $1,56(1,29)$ & 2,26 & 0,09 \\
Ineficácia da punição física & $1,05(1,05)$ & $1,00(0,79)$ & 0,43 & 0,73 \\
Limites para a punição física & $0,50(0,68)$ & $0,35(0,58)$ & 0,93 & 0,43 \\
Necessidade da punição física & $0,70(0,97)$ & $0,35(0,58)$ & 1,08 & 0,37 \\
\hline g.l. =1
\end{tabular}

Com o objetivo de avaliar as diferenças nas frequências médias das crenças sobre a privação de privilégios/castigos entre os dois grupos foi realizada uma Análise de Covariância (ANCOVA) com as frequências médias para cada categoria. As variáveis idade da mãe e idade da criança foram incluídas como covariantes. A Tabela 3 apresenta a média, o desvio padrão, o valor de $\mathrm{F}$ e o nível de significância das categorias de crenças sobre a privação de privilégios nos dois grupos. 
Tabela 3

Incidência Média, Desvio Padrão, Valor de F e Nivel de Significância do Total de Crenças Maternas Sobre a Privação de Privilégios Mencionadas pelos Dois Grupos

\begin{tabular}{lcccc}
\hline & $\begin{array}{c}\text { Grupo NSE-B } \\
\text { Média (D.P) }\end{array}$ & $\begin{array}{c}\text { Grupo NSE-M } \\
\text { Média (D.P) }\end{array}$ & $\mathrm{F}$ & $\mathrm{p}<$ \\
\hline Eficácia da privação & $1,85(0,74)$ & $2,10(0,96)$ & 3,10 & 0,03 \\
Ineficácia da privação & $0,10(0,30)$ & $0,25(0,63)$ & 2,25 & 0,09 \\
Limites para a privação & $0,55(0,60)$ & $0,45(0,60)$ & 0,19 & 0,90 \\
Necessidade da privação & $0,35(0,58)$ & $0,10(0,30)$ & 0,96 & 0,41 \\
\hline g.l. $=1$ & & & &
\end{tabular}

A única categoria que apresentou diferença estatisticamente significativa entre os grupos foi eficácia da privação de privilégios ( $F$ $=3,10 ; p=0,03)$. As médias indicam maior frequência desta categoria de crença no grupo NSE-M $(M=2,10$; $D P=0,96)$, em comparação ao grupo NSE-B $(M=1,85 ; \mathrm{DP}=0,74)$. Nas demais categorias de crenças sobre a privação de privilégios, as frequências de respostas das mães dos dois grupos foram semelhantes.

\section{Discussão}

O presente estudo comparou as crenças de mães de diferentes NSE quanto ao uso de práticas de socialização coercitivas. Ao contrário do que se esperava, os resultados do estudo não confirmaram a hipótese de que as mães de NSE-B relatariam mais crenças favoráveis às práticas coercitivas quando comparadas às mães de NSE-M, o que indica que, do ponto de vista do que pensam as mães sobre as práticas coercitivas, o NSE não parece produzir nenhum impacto significativo. Assim, de forma contrária aos achados de Cappa e Khan (2011) e de Giles-Sims e Lockhart (2005), que demonstravam relação entre as crenças sobre a legitimidade e eficácia da punição física e o nível socioeconômico, os achados do presente estudo não corroboraram essa hipótese. Por outro lado, outros estudos, da mesma forma, têm indicado a possibilidade de ausência de relação entre as duas variáveis. Wiehe (1990) e Grogan-Kaylor e Otis (2007), por exemplo, verificaram que características socioeconômicas das mães, tais como a idade, o nível de escolaridade, o tipo de bairro no qual reside e a renda, não possuíam relação com a presença ou a ausência de crenças que legitimam o uso da punição física. Dessa forma, outras variáveis, tais como a cultura, podem ser mais importantes para a compreensão das crenças parentais.

A ausência de influência do NSE das participantes sobre as crenças a respeito da punição física, verificada neste estudo, permite levantar a hipótese de que as variáveis responsáveis pela formação dessas crenças estão presentes e afetam os dois grupos de forma 
semelhante. $O$ crescente interesse dos meios de comunicação em divulgar as desvantagens e as consequências negativas da punição física para o desenvolvimento infantil e a amplitude com que tais informações têm sido disseminadas, podem estar produzindo efeitos benéficos sobre os sistemas de crenças de pais e mães. Assim, é possível que ambos os grupos avaliados neste estudo tenham sido afetados similarmente. Isso explicaria as semelhanças no relato verbal das mães de NSE baixo e de NSE médio/alto. Contudo, ainda resta compreender porque esses efeitos não se traduzem em práticas de socialização igualmente mais funcionais, eficazes e positivas, na medida em que a literatura tem mostrado que o uso da punição física e de outras estratégias coercitivas ainda são frequentes no repertório dos pais (Alvarenga \& Piccinini, 2001; Bem \& Wagner, 2006; Doe, 2000; Gracia \& Herrero, 2008; Malhi \& Ray, 2004). É importante destacar que o estudo de Carmo e Alvarenga (2012), que avaliou as práticas de socialização utilizadas pelas mesmas participantes que compuseram os dois grupos analisados no presente estudo, revelou que as mães de NSE baixo relataram utilizar a punição física com uma frequência significativamente mais alta do que as mães de NSE médio/alto.

Esta possível dissonância entre as crenças maternas, que parecem assimilar e incorporar o discurso sobre os efeitos deletérios das práticas coercitivas, e as práticas maternas, aparentemente menos vulneráveis a esses enunciados, pode ser, pelo menos em parte, explicada pela ausência de estratégias efetivas de ampliação do repertório de práticas de socialização de pais e mães. É possível que campanhas e outras iniciativas voltadas para práticas de socialização infantil no contexto familiar estejam falhando na medida em que focalizam quase que exclusivamente as práticas que devem ser evitadas pelos pais (ex. bater ou humilhar a criança) e não nas estratégias alternativas que poderiam ser adotadas (ex. estabelecer regras claras, negociar ou raciocinar junto com a criança sobre os comportamentos inadequados e as possíveis consequências). Nesse sentido, os pais estariam se tornando progressivamente mais hábeis para descrever e identificar os efeitos prejudiciais das práticas coercitivas, porém, não estariam aprimorando seu repertório de práticas assertivas e eficazes para favorecer o desenvolvimento socioemocional de seus filhos. Assim, os pais acabariam por adotar esse tipo de estratégia, mesmo sabendo de suas desvantagens, por não dispor de um repertório alternativo. Dessa forma, é fundamental que intervenções com esse objetivo, mesmo aquelas que possuem apenas caráter informativo, incluam orientações claras e objetivas sobre estratégias alternativas à coerção, que instrumentalizem pais e mães para lidar de maneira eficaz com conflitos em seu cotidiano com os filhos. 
Ainda assim, é importante destacar que a coleta das informações, sobre as crenças maternas, foi realizada através de entrevistas, que estão associadas à desejabilidade social. É possível que a disseminação de informações sobre as desvantagens das práticas coercitivas, associadas à desejabilidade social, tenham diminuído a probabilidade das participantes relatarem que acreditavam na eficácia e na necessidade das práticas coercitivas. É preciso considerar também o fato de que a escala utilizada para avaliar o NSE das famílias, além de não considerar diretamente a renda como critério de classificação, não foi construída para a população brasileira, embora tenha passado por um processo de adaptação. Nesse sentido, o instrumento de avaliação do NSE certamente tem limitações e repercutiu na caracterização dos dois grupos de comparação, podendo também estar, em certa medida, relacionado às semelhanças verificadas nas crenças.

Outro aspecto que deve ser considerado em futuros estudos para melhor compreensão das relações entre o NSE e as crenças parentais é a avaliação do NSE da família de origem. Em um estudo que se propôs a avaliar possíveis diferenças a respeito do apoio ao uso de estratégias parentais coercitivas, como as surras, e a valorização da obediência, considerando o NSE da família de origem e o NSE atual, em uma amostra nacionalmente representativa dos Estados Unidos, Friedson (2016) verificou que apoiar o uso de surras e valorizar a obediência por parte da criança, estiveram negativamente correlacionados ao NSE da família de origem dos pais da criança, até mesmo controlando o efeito do NSE atual. Esse achado indica que, quanto mais baixo o NSE da família de origem, mais o adulto irá valorizar e apoiar práticas coercitivas como estratégias de regulação do comportamento de crianças. De acordo com o autor, é provável que crescer em um ambiente com restrições socioeconômicas contribua para o aprendizado de valores, crenças e expectativas sobre como interagir com o entorno social que são difíceis de serem alterados ao longo da vida do indivíduo e que, em geral, envolvem o uso da força para controle e obediência por parte da criança.

Com relação ao impacto do NSE sobre as crenças relacionadas à privação de privilégios/castigo, foi encontrada uma única diferença entre os grupos, que indicou que as mães do NSE-M relataram mais crenças sobre a eficácia da privação de privilégios. A literatura da Psicologia do Desenvolvimento revisada não oferece argumentos que expliquem essa relação. A hipótese levantada para compreender esse resultado relaciona-se à possibilidade de o grupo NSE-M apresentar maior variabilidade de práticas parentais quando comparado ao grupo NSE-B (Carmo \& Alvarenga, 2012). Mães de NSE-M, ao utilizarem com mais frequência outras práticas para regulação do comportamento dos filhos, além da punição física, teriam maior probabilidade de ficar sob controle das consequências dessas 
práticas, e assim, elaborar regras sobre sua utilização. Nesse sentido, descrever a privação de privilégios como uma estratégia eficaz seria resultado da utilização de tal prática com maior frequência, e da observação de sua eficácia no controle do comportamento infantil indesejado. Entretanto, essa é apenas uma hipótese, sendo necessário que mais estudos avaliem as relações entre esse tipo de prática e o NSE das famílias de forma que essa hipótese seja confirmada ou não.

Em resumo, os achados que apontaram para a ausência de diferenças entre as crenças das mães dos diferentes grupos destacam um aspecto positivo, visto que mães que vivem em condições menos favoráveis do ponto de vista socioeconômico não se encontram em uma situação mais vulnerável do que aquelas que dispõem de mais recursos. Porém, considerando-se os achados de outros estudos que apontam consistentemente para o uso mais frequente de práticas coercitivas entre mães de NSE baixo, é necessário que novas investigações busquem esclarecer essa aparente discrepância, bem como as relações entre as crenças e as práticas de socialização parentais, especialmente a privação de privilégios.

Apesar do pequeno número de casos, que não permitiu uma análise mais refinada a respeito das crenças maternas e suas relações com o NSE e de não ter investigado o NSE da família de origem, o presente estudo investigou as crenças a respeito de uma prática ainda pouco estudada pela literatura da Psicologia do Desenvolvimento, nacional e internacional, a privação de privilégios. A elucidação desses processos contribuirá para uma melhor compreensão das práticas utilizadas pelos pais para socializar os filhos, e poderá resultar em ações mais efetivas para a promoção do processo de socialização infantil no contexto familiar.

\section{Referências}

Alvarenga, P., Magalhães, M. O., \& Gomes, Q. S. (2012). Relações entre práticas educativas maternas e problemas de externalização em pré-escolares. Estudos de Psicologia (Campinas), 29(1), 33-42. doi: 10.1590/S0103166X2012000100004

Alvarenga, P., \& Palma, E. (2012). Parentalidade e comportamento antissocial infantil. In C. A. Piccinini \& P. Alvarenga (Eds.), Maternidade e paternidade: a parentalidade em diferentes contextos (pp. 233-257). São Paulo, SP: Casa do Psicólogo.

Alvarenga, P., \& Piccinini, C. A. (2001). Práticas educativas maternas e problemas de comportamentos em pré-escolares. Psicologia: Reflexão E Crítica, 14(3), 449-460. doi:10.1590/S010279722001000300002 
Bardin, L. (2011). Análise de Conteúdo (Trad. L. A. Reto \& A. Pinheiro). Lisboa: Edições 70. (Trabalho original publicado em 1977)

Bayer, J. K., Ukoumunne, O. C., Mathers, M., Wake, M., Abdi, N., \& Hiscock, H. (2012). Development of children's internalising and externalising problems from infancy to five years of age. Australian and New Zealand Journal of Psychiatry, 46(7), 659668. doi: $10.1177 / 0004867412450076$

Bem, L. A., \& Wagner, A. (2006). Reflexões sobre a construção da parentalidade e o uso de estratégias educativas em famílias de baixo nível socioeconômico. Psicologia Em Estudo, 11(1), 6371. doi: 10.1590/S1413-73722006000100008

Bor, W., \& Sanders, M. R. (2004). Correlates of self-reported coercive parenting of preschool-aged children at high risk for the development of conduct problems. The Australian and New Zealand Journal of Psychiatry, 38(9), 738-745. doi: 10.1111/j.1440-1614.2004.01452.x

Bornstein, M. H., \& Bradley, R. H. (2003). Socioeconomic status, parenting, and child development: an introduction. In $\mathrm{M} . \mathrm{H}$. Bornstein, \& R. H. Bradley (Eds.), Socioeconomic Status, Parenting, and Child Development (pp. 1-10). New Jersey: Lawrence Erlbaum Associates.

Bradley, R. H., \& Corwyn, R. F. (2002). Socioeconomic status and child development. Annual Review of Psychology, 53, 371- 399. doi: 10.1146/annurev.psych.53.100901.135233

Cappa, C., \& Khan, S. M. (2011). Understanding caregivers' attitudes towards physical punishment of children: Evidence from 34 lowand middle-income countries. Child Abuse \& Neglect, 35(12), 1009-1021. doi: 10.1016/j.chiabu.2011.10.003

Carmo, P. H. B. (2009). Práticas educativas coercitivas e crenças sobre a coerção em mães de diferentes níveis socioeconômicos. (Dissertação de Mestrado). Retrieved from http://www.pospsi.ufba.br/Paulo_Henique_Barbosa.pdf

Carmo, P. H. B., \& Alvarenga, P. (2012). Práticas educativas coercitivas de mães de diferentes níveis socioeconômicos. Estudos de Psicologia (Natal), 17(2), 191-197. doi: 10.1590/S1413-294X2012000200001

Doe, S. S. (2000). Cultural factors in child maltreatment and domestic violence in Korea. Children and Youth Services Review, 22(3-4), 231-236. doi: 10.1016/S01907409(00)00077-3

Friedson, M. (2016). Authoritarian parenting attitudes and social origin: The multigenerational relationship of socioeconomic position to childrearing values. Child Abuse \& Neglect, 51(1), 263-275. doi: 10.1016/j.chiabu.2015.10.001 
Giles-Sims, J., \& Lockhart, C. (2005). Culturally shaped patterns of disciplining children. J ournal of Family Issues, 26(2), 196-218. doi: 10.1177/0192513X04270414

Gracia, E., \& Herrero, J. (2008). Is it considered violence? The acceptability of physical punishment of children in Europe. Journal of Marriage and Family, 70(1), 210-217. doi: 10.1111/j.1741-3737.2007.00472.x

Grogan-Kaylor, A., \& Otis, M. D. (2007). The predictors of parental use of corporal punishment. Family Relations, 56(1), 80-91. doi: http://dx.doi.org/10.1111/j.1741-3729.2007.00441.x

Guerra, N. G., Huesmann, L. R., Tolan, P. H., Van Acker, R., \& Eron, L. D. (1995). Stressful events and individual beliefs as correlates of economic disadvantage and aggression among urban children. Journal of Consulting and Clinical Psychology, 63(4), 518-528. doi: 10.1037/0022-006X.63.4.518

Hollingshead, A. B. (1975). Four factor index of social status. (Manuscrito não publicado).

Jansen, P. W., Raat, H., Mackenbach, J. P., Hofman, A., Jaddoe, V. W., Bakermans-Kranenburg, J. M., ... Tiemeier, H. (2012). Early determinants of maternal and paternal harsh discipline: The generation $\mathrm{R}$ study. Family Relations, 61(2), 253-270. doi: 10.1111/j.1741-3729.2011.00691.x

Knutson, J. F., DeGarmo, D. S., Koeppl, G., \& Reid, J. B. (2005). Care neglect, supervisory neglect, and harsh parenting in the development of children's aggression: a replication and extension. Child Maltreatment, 10(2), 92-107. doi: $10.1177 / 1077559504273684$

Kobarg, Sachetti, V. A. R., \& Vieira, M. L. (2006). Valores e crenças parentais: reflexões teóricas. Revista Brasileira Crescimento $\mathrm{E}$ Desenvolvimento Humano, 16(2), 96-102. Retrieved from http: // pepsic. bvsalud.org/pdf/rbcdh/v16n2/10.pdf

Lerner, R. M. (2003). What are SES effects effects of? A Developmental Systems perspective. In M. H. Bornstein \& R. H. Bradley (Eds.), Socioeconomic Status, Parenting, and Child Development (pp. 231-255). New Jersey: Lawrence Erlbaum Associates.

Lins, T., Alvarenga, P., Paixão, C., Almeida, E., \& Costa, H. (2012). Problemas externalizantes e agressividade infantil: uma revisão de estudos brasileiros. Arquivos Brasileiros de Psicologia, 64(3), 57-75. Retrieved

from http: //www. redalyc. org/articulo. oa?id $=229025830010$

Malhi, P., \& Ray, M. (2004). Prevalence and correlates of corporal punishment among adolescents. Studia Psychologica, 46(3), 219-227. Retrieved from http://www.psychologia.sav.sk/sp/index.php?id=abstract\&numi $d=349$ 
Mistry, R. S., Vandewater, E. A., Huston, A. C., \& McLoyd, V. C. (2002). Economic well-being and children's social adjustment: the role of family process in an ethnically diverse low-income sample. Child Development, 73(3), 935-951. doi: 10.1111/1467-8624.00448

Moura, M. L. S., Ribas Jr., R. C., Piccinini, C. A., Bastos, A. C. S., Magalhães, C. M. C., Vieira, M. L., ... Silva, A. K. (2004). Conhecimento sobre desenvolvimento infantil em mães primíparas de diferentes centros urbanos do Brasil. Estudos de Psicologia (Natal), 9(3), 421-429. doi:10.1590/S1413294X2004000300004

Passini, C. M., Pihet, S., \& Favez, N. (2014). Assessing specific discipline techniques: A mixed-methods approach. Journal of Child and Family Studies, 23(8), 1389-1402. doi: 10.1007/s10826-013-9796-0

Ribas Jr., R. C., Moura, M. L. S., \& Bornstein, M. H. (2003). Socioeconomic status in Brazilian psychological research: II. socioeconomic status and parenting knowledge. Estudos de Psicologia (Natal), 8(3), 385-392. doi:10.1590/S1413294X2003000300005

Ribas Jr., R. C., Moura, M. L. S., Soares, I. D., Gomes, A. A. N., \& Bornstein, M. H. (2003). Socioeconomic status in Brazilian psychological research: I. validity, measurement, and application. Estudos de Psicologia (Natal), 8(3), 375-383. doi: 10.1590/S1413-294X2003000300004

Shor, R. (2000). Child maltreatment: Differences in perceptions between parents in low income and middle income neighborhoods. British Journal of Social Work, 30(2), 165-178. doi: 10.1093/bjsw/30.2.165

Socolar, R. S., Savage, E., Keyes-Elstein, L., Evans, H. (2005). Factors that affect parental disciplinary practices of children aged 12 to 19 months. Southern Medical J ournal, 98(12), 11811191. doi: 10.1097/01.smj.0000190177.12387.07

Theunissen, M. H. C., Vogels, A. G. C., \& Reijneveld, S. A. (2015). Punishment and reward in parental discipline for children aged 5 to 6 years: prevalence and groups at risk. Academic Pediatrics, 15(1), 96-102. doi:10.1016/j.acap.2014.06.024

Tudge, J. R. H., \& Frizzo, G. B. (2002). Classificação baseada em Hollingshead do nível sócio-econômico das famílias do estudo longitudinal de Porto Alegre: da gestação à escola. Manuscrito Não Publicado.

Weber, L. N. D., Viezzer, A. P., \& Brandenburg, O. J. (2004). O uso de palmadas e surras como prática educativa. Estudos de Psicologia (Natal), 9(2), 227-237. doi:10.1590/S1413294X2004000200004 
Wiehe, V. (1990). Religious influence on parental attitudes toward the use of corporal punishment. Journal of Family Violence, 5(2), 173-186. doi: 10.1007/BF00978518

\section{Endereço para correspondência \\ Paulo Henrique Barbosa do Carmo}

Faculdade Anísio Teixeira

Rua J uracy Magalhães, 222, Ponto Central, CEP 44032-620, Feira de Santana - BA, Brasil

Endereço eletrônico: phbc@oi.com.br

Patrícia Alvarenga

Universidade Federal da Bahia

Rua Aristides Novis, Estrada de São Lázaro, 197, CEP 40210-730, Salvador - BA, Brasil

Endereço eletrônico: palvarenga66@gmail.com

Taiane Costa de Souza Lins

Universidade Federal da Bahia

Rua Aristides Novis, Estrada de São Lázaro, 197, CEP 40210-730, Salvador - BA, Brasil

Endereço eletrônico: lins.taiane@gmail.com

Recebido em: 07/04/2016

Reformulado em: 20/07/2016

Aceito para publicação em: 22/07/2016

\section{Notas}

* Mestre em Psicologia pela Universidade Federal da Bahia (UFBA), especialista em Psicoterapia Comportamental (Universidade de São Paulo) e professor na Faculdade Anísio Teixeira.

** Doutora em Psicologia pela Universidade Federal do Rio Grande do Sul (UFRGS) e professora associada do Instituto de Psicologia da Universidade Federal da Bahia (UFBA).

*** Mestre e doutoranda em Psicologia pela Universidade Federal da Bahia (UFBA) e professora na Faculdade Anísio Teixeira. 